\title{
Serum NT-proBNP and TUG1 as novel biomarkers for elderly hypertensive patients with heart failure with preserved ejection fraction
}

\author{
SHUANG ZHANG, RIZE JIN and BI LI \\ Ultrasonic Department, The First Affiliated Hospital of Jinzhou Medical University, \\ Jinzhou, Liaoning 121000, P.R. China
}

Received July 23, 2020; Accepted December 8, 2020

DOI: $10.3892 /$ etm.2021.9874

\begin{abstract}
Patients with heart failure with preserved ejection fraction (HFPEF) account for $~ 50 \%$ of all cases of heart failure and their clinical prognosis is poor. The present study attempted to investigate the diagnostic value of circulating long non-coding RNA taurine upregulated gene 1 (TUG1) for HFPEF in subjects with hypertension. Between January 2017 and January 2019, 80 aged/elderly hypertensive patients with or without HFPEF were recruited for the present study. The concentration of N-terminal pro-brain natriuretic peptide (NT-proBNP) in the serum was measured using ELISA and TUG1 expression levels were determined using reverse transcription-quantitative PCR. Echocardiography was used for the determination of cardiac function. The results indicated that the levels of NT-proBNP and TUG1 were increased in the serum of hypertensive patients with HFPEF. Pearson analysis demonstrated that NT-proBNP and TUG1 were positively correlated with the left atrial diameter and negatively correlated with the ratio of the peak flow velocity in the early diastolic phase to the peak flow velocity in the late diastolic phase. In addition, a positive correlation was confirmed between TUG1 and NT-proBNP levels. Receiver operating characteristic curve analysis demonstrated that TUG1 and NT-proBNP were useful biomarkers for the diagnosis of HFPEF. In conclusion, it was observed that NT-proBNP and TUG1 were increased in the serum of hypertensive patients with HFPEF. Furthermore, TUG1 and NT-proBNP were indicated to be useful plasma biomarkers for the diagnosis of HFPEF.
\end{abstract}

Correspondence to: Dr Shuang Zhang, Ultrasonic Department, The First Affiliated Hospital of Jinzhou Medical University, 2 Section, 5 Renmin Street, Jinzhou, Liaoning 121000, P.R. China E-mail: zhangshuang_jmu@163.com

Key words: heart failure with preserved ejection fraction, $\mathrm{N}$-terminal pro-brain natriuretic peptide, taurine upregulated gene 1

\section{Introduction}

Heart failure (HF) refers to a syndrome in which venous reflux is normal and cardiac output is reduced due to primary cardiac damage, which cannot meet the requirements of tissue metabolism. Pulmonary circulation and/or systemic circulation congestion and inadequate tissue perfusion are the major characteristics of clinical HF; thus, it is also called congestive HF and is the final stage of heart disease due to various causes (1). With an aging population, HF-associated morbidity and mortality have exhibited an upward trend in the United States and globally (2). It has been indicated that certain patients with HF still maintained a relatively normal left ventricular ejection fraction (LVEF), which is known as HF with preserved ejection fraction (HFPEF) (3). Patients with HFPEF accounted for $\sim 50 \%$ of all cases of HF and their clinical prognosis is poor (4). Echocardiography is a non-invasive examination method with simple operation, so it is widely adopted in the clinic to assess heart function. However, HFPEF cannot always be detected at the early stages, whilst early diagnosis is important for saving the life of affected patients and easing the disease burden. With the fast development in biomedical technology, biomarker-based determination has received increasing attention. Plasma pro-brain natriuretic peptide (BNP), a hormonal biologically active substance secreted by left ventricular wall cells, is the major serum marker to evaluate the severity of HF (5).

As a class of non-coding transcripts, long non-coding RNAs (lncRNAs) are $>200 \mathrm{nt}$ in length and regulate gene expression at epigenetic, transcriptional and post-transcriptional levels $(6,7)$. Aberrant expression levels of various IncRNAs in the serum have recently been utilized as diagnostic biomarkers, e.g. for cardiac diseases (8). For instance, long-intergenic noncoding RNA predicting cardiac remodeling was able to predict survival in patients with HF (9). HOX transcript antisense RNA is an essential mediator of acute myocardial infarction (10). Antisense non-coding RNA in the INK4 locus is of important diagnostic value for in-stent restenosis (11). The lncRNA taurine upregulated gene 1 (TUG1) is a newly discovered lncRNA, which was originally detected in mouse retinal cells treated with taurine- $\beta$ (12). However, the molecular function of TUG1 in patients with 
HFPEF and hypertension has remained elusive. In the present study, the role of circulating TUG1 in the diagnosis of HFPEF in subjects with hypertension was investigated.

\section{Materials and methods}

Participants and samples. Between January 2017 and January 2019, 80 aged/elderly hypertensive patients (Table I) with HFPEF were recruited as the 'observation group' at The First Affiliated Hospital of Jinzhou Medical University (Jinzhou, China). Written informed consent was provided by all study participants. The present study was approved by the ethics committee of The First Affiliated Hospital of Jinzhou Medical University. The diagnostic criteria for HFPEF were according to the European Society of Cardiology guidelines for the diagnosis and treatment of acute and chronic HF from 2016 (13): i) Signs or symptoms of HF; ii) elevated levels of BNPs (BNP>35 pg/ml and/or NT-proBNP>125 pg/ml); iii) evidence of cardiac structural alterations (left atrial volume index $>34 \mathrm{ml} / \mathrm{m}^{2}$ or a left ventricular mass index $\geq 115 \mathrm{~g} / \mathrm{m}^{2}$ for males and $\geq 95 \mathrm{~g} / \mathrm{m}^{2}$ for females) or functional features of diastolic dysfunction [ratio of early diastolic mitral flow velocity to early diastolic mitral ring velocity $\left(E / e^{\prime}\right) \geq 13$ and a mean E' septal and lateral wall $<9 \mathrm{~cm} / \mathrm{sec}$; iv) normal or slightly abnormal LVEF (>50\%) and the left ventricle was not enlarged; and v) left ventricular diastolic dysfunction. The patients in the observation group were included if they fulfilled the following criteria: i) Age, 60-75 years; ii) systolic blood pressure $>150 \mathrm{mmHg}$ and normal diastolic blood pressure; iii) conformed to the diagnostic criteria of HFPEF. Patients were excluded under the following circumstances: i) LVEF $<50 \%$; ii) chronic obstructive pulmonary disease, valvular heart disease, hypertrophic cardiomyopathy, restrictive cardiomyopathy, pericardial disease or diabetes mellitus; iii) hematological and neoplastic disorders or autoimmune diseases; iv) severe liver or kidney dysfunction. Furthermore, 80 aged/elderly hypertensive patients (age, 60-75 years; systolic blood pressure $>150 \mathrm{mmHg}$; not combined with any other diseases) were enrolled as a control group. Furthermore, according to the New York Heart Association (NYHA) cardiac function classification (14), the patients in the observation group were divided into four groups with NYHA I-IV, respectively (Table II). After overnight fasting, $5 \mathrm{ml}$ peripheral blood samples were collected from the brachial vein of each patient. Whole blood was centrifuged at $1,500 \mathrm{xg}$ for $5 \mathrm{~min}$ at room temperature and the supernatant was immediately frozen and stored at $-80^{\circ} \mathrm{C}$ for further analysis.

Measurement of NT-proBNP. The concentration of NT-proBNP in the serum of each participant was determined using an ELISA kit (cat. no. DY3604-05; R\&D Systems, Inc.) according to the manufacturer's protocol. A microplate reader was used to determine the absorbance at $450 \mathrm{~nm}$.

Assessment of cardiac function. The patients were kept in a left lateral position for echocardiography examinations. M-mode echocardiography was used for the determination of the left ventricular end diastolic diameter (LVEDD), left atrial diameter (LAD), left ventricular posterior wall thickness and interventricular septal thickness (LVPWT), interventricular septal thickness (IVS). Pulse Doppler echocardiography was adopted to measure the ratio of the peak flow velocity in the early diastolic phase to the peak flow velocity in the late diastolic phase (E/A).

Reverse transcription-quantitative (RT-q)PCR analysis. Total RNA in the serum of all paticipants was isolated using TRIzol ${ }^{\circledast}$ reagent (Invitrogen; Thermo Fisher Scientific, Inc.) following the manufacturer's protocol. Complementary DNA synthesis was performed using the TaqMan MicroRNA RT kit (Applied Biosystems; Thermo Fisher Scientific, Inc.). Total RNA was reverse transcribed into cDNA at $37^{\circ} \mathrm{C}$ for $2 \mathrm{~h}$, at $95^{\circ} \mathrm{C}$ for $15 \mathrm{~min}$ and then was cooled on ice. Real-time PCR was performed using SYBR ${ }^{\circledR}$ Premix Ex Taq ${ }^{\mathrm{TM}}$ II (Takara Bio, Inc.). The relative expression was analyzed using the $2^{-\Delta \Delta C q}$ method (15) with normalization to GAPDH. The primer sequences used for qPCR were shown as follows: TUG1 forward, 5'-TAGCAGTTCCCCAATCCTTG-3' and reverse, 5'-CACAAATTCCCATCATTCCC-3' and GAPDH forward, 5'-GGGAGCCAAAAGGGTCAT-3' and reverse, 5'-GTGGTT TGAGGGCTCTTACTCCTT-3'. The qPCR thermocycling conditions used were as follows: Initial denaturation at $95^{\circ} \mathrm{C}$ for $10 \mathrm{~min}$, followed by denaturation at $94^{\circ} \mathrm{C}$ for $15 \mathrm{sec}$, annealing at $59^{\circ} \mathrm{C}$ for $30 \mathrm{sec}$ and extension at $70^{\circ} \mathrm{C}$ for $30 \mathrm{sec}$, for 40 cycles.

Statistical analysis. Statistical analyses were performed using SPSS software (version no. 21.0; IBM Corp.).All measurement data were expressed as the mean \pm standard deviation. Differences were calculated using Student's t-test or one-way ANOVA followed by Tukey's post hoc test. The correlation analysis was performed using Spearman's correlation method. The diagnostic efficacy of the plasma indexes was determined using receiver operating characteristics (ROC) curve analyses. $\mathrm{P}<0.05$ was considered to indicate statistical significance.

\section{Results}

NT-proBNP concentration is increased in serum of hypertensive patients with HFPEF. The general demographic and clinicopathological data of all patients are listed in Table I. There were no significant differences in clinical materials of these two groups including age, gender and some risk factors $(P>0.05)$. First, the NT-proBNP concentration in the serum of patients was measured using ELISA. The results demonstrated that the NT-proBNP concentration was significantly upregulated in the observation group as compared with that in the control group (Fig. 1A). Furthermore, the NT-proBNP concentration was significantly increased with the severity degree of HF (Fig. 1B). Spearman's rank correlation analysis demonstrated that the NT-proBNP concentration was positively correlated with the LAD and negatively correlated with the E/A (Fig. 1C and D, respectively).

Serum TUG1 is elevated in hypertensive patients with HFPEF. The levels of TUG1 in the serum of patients were then assessed using RT-qPCR. The levels of TUG1 were significantly enhanced in the observation group in comparison with those in the control group (Fig. 2A). Furthermore, TUG1 expression levels were significantly augmented with the 
Table I. Clinical characteristics of patients in the control and observation groups.

\begin{tabular}{lccc}
\hline Variable & Control group $(\mathrm{n}=80)$ & Observation group $(\mathrm{n}=80)$ & P-value \\
\hline Age (years) & $68.24 \pm 6.13$ & $66.87 \pm 7.44$ & 0.125 \\
Male sex $(\%)$ & $48(60 \%)$ & $46(57.5 \%)$ & 0.748 \\
BMI $\left(\mathrm{kg} / \mathrm{m}^{2}\right)$ & $26.21 \pm 2.58$ & $25.78 \pm 2.97$ & 0.528 \\
Smoking $(\%)$ & $50(62.5 \%)$ & $49(61.25 \%)$ & 0.871 \\
History of hypertension (years) & $10.11 \pm 2.81$ & $11.36 \pm 1.44$ & 0.215 \\
Blood glucose $(\mathrm{mmol} / \mathrm{l})$ & $6.25 \pm 1.13$ & $6.12 \pm 1.44$ & 0.853 \\
Serum creatinine $(\mu \mathrm{mol} / \mathrm{l})$ & $82.56 \pm 10.54$ & $75.58 \pm 9.38$ & 0.521 \\
Hyperlipemia $(\%)$ & $16(25 \%)$ & $24(30 \%)$ & 0.479 \\
NYHA & & 34 & 26 \\
II & $/$ & 20 \\
III & $/$ & & 34 \\
IV & $/$ & & \\
\hline
\end{tabular}

NYHA, New York Heart Association classification; BMI, body mass index.

Table II. Echocardiographic indexes in the control and observation groups.

\begin{tabular}{lccr}
\hline Variable & Control group $(\mathrm{n}=80)$ & Observation group $(\mathrm{n}=80)$ & P-value \\
\hline LVEDD (mm) & $50.56 \pm 10.52$ & $51.32 \pm 9.78$ & 0.425 \\
LAD (mm) & $38.56 \pm 7.63$ & $48.21 \pm 5.96$ & 0.001 \\
LVEF (\%) & $0.64 \pm 0.08$ & $0.63 \pm 0.05$ & 0.358 \\
LVPWT (mm) & $9.26 \pm 1.38$ & $9.19 \pm 1.71$ & 0.481 \\
IVS (mm) & $9.87 \pm 1.85$ & $9.17 \pm 1.31$ & 0.412 \\
E/A & $1.67 \pm 0.81$ & $0.89 \pm 0.47$ & 0.001
\end{tabular}

LVEDD, left ventricular end diastolic diameter; LAD, left atrial diameter; LVPWT, left ventricular posterior wall thickness; IVS, interventricular septal thickness; E/A, ratio of the peak flow velocity in the early diastolic phase to the peak flow velocity in the late diastolic phase.

increase in the severity degree of HF (Fig. 2B). Spearman's correlation analysis demonstrated that TUG1 was positively correlated with the LAD and negatively correlated with the E/A (Fig. 2C and D, respectively).

TUG1 and NT-proBNP are useful diagnostic biomarkers for HFPEF in a hypertensive population. A positive correlation between the serum levels of TUG1 and NT-proBNP was confirmed (Fig. 3A). ROC curve analysis suggested that TUG1 and NT-proBNP are useful biomarkers for the diagnosis of HFPEF among subjects with hypertension, with area under the curve (AUC) values of 0.73 (95\% CI, 0.67-0.79; cut-off value, 0.43 ) and 0.76 (95\% CI: 0.71-0.85; cut-off value, 0.45$)$. In addition, the combination of the two parameters slightly improved the diagnostic power, with an AUC of 0.83 (95\% CI, 0.74-0.89; cut-off value, 0.48).

\section{Discussion}

HFPEF is caused by decreased cardiac smooth muscle relaxation during the process of left ventricular diastole. When HFPEF occurs, the myocardial compliance is impaired and the diastolic capacity of the ventricular muscle is weakened, resulting in decreased filling function of the left ventricle during the diastolic period and decreased output per stroke, which then leads to an increase of the filling pressure at the end of the diastolic period of the left ventricle, finally inducing HF (16). HFPEF has numerous causes and the most common etiology is hypertension in the elderly (17). By determining the two-dimensional ultrasonic diagnostic index, the size of each atrioventricular cavity of the heart may be rapidly measured, which is helpful for the diagnosis of HFPEF. Blood flow Doppler and tissue Doppler are sensitive for the measurement of mitral valve diastole, systolic blood flow velocity and mitral annulus root movement velocity (18). In the present study, it was observed that the LAD was significantly thicker in hypertensive patients with HFPEF compared with that in the control group of patients with hypertension. Furthermore, the E/A was markedly decreased in these patients. NT-proBNP is a precursor of BNP, which is mainly synthesized and secreted by ventricular myocytes. It may reflect the functions of left ventricular systole and remodeling and is of important diagnostic value for HF (19). The present results suggested that the concentration of NT-proBNP was significantly elevated in hypertensive patients with HFPEF compared with that in the control group of patients with hypertension. The NYHA 

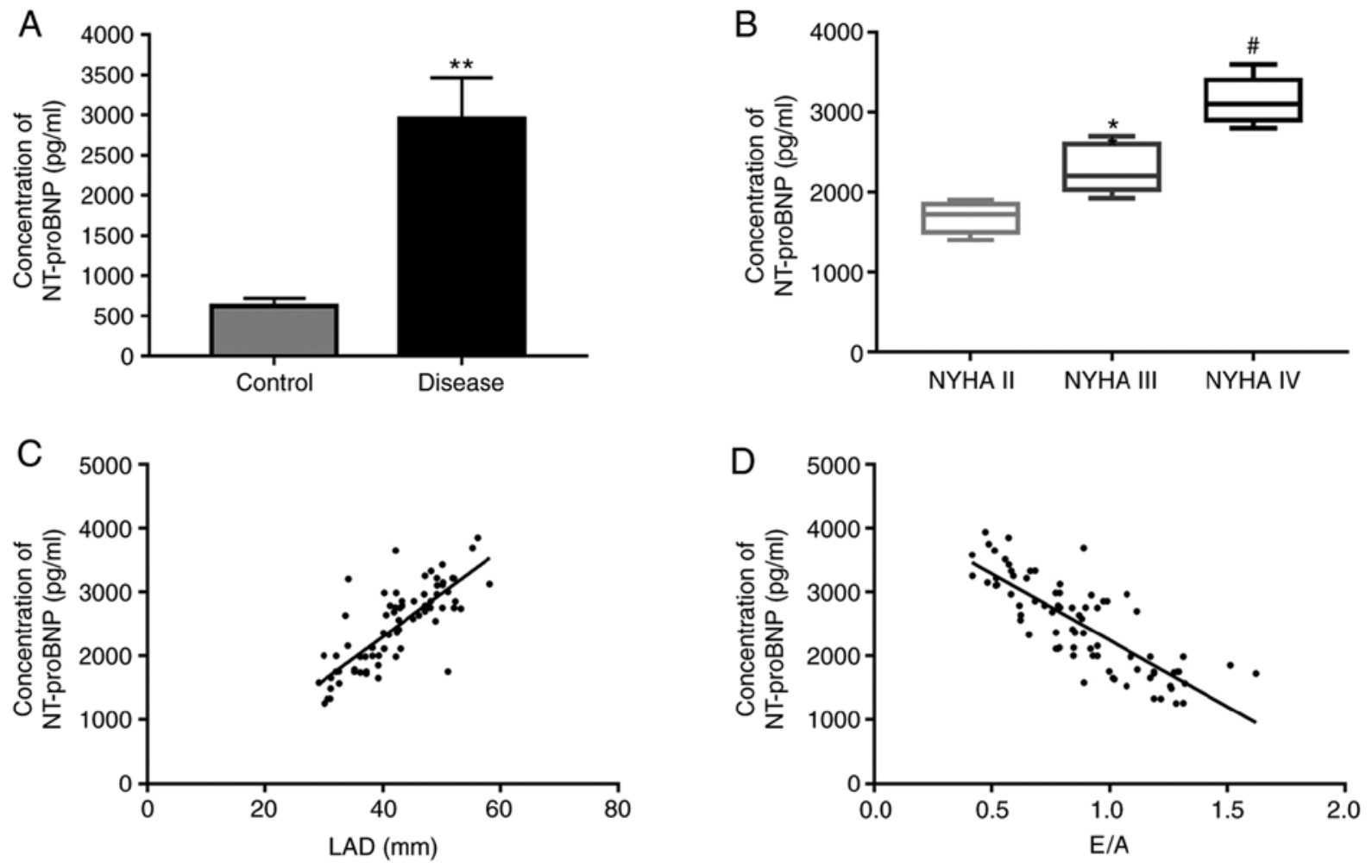

Figure 1. Serum NT-proBNP is increased in hypertensive patients with heart failure with preserved ejection fraction. (A) The NT-proBNP concentration was significantly upregulated in the observation group compared with that in the control. (B) The NT-proBNP concentration was significantly increased in the NYHA III vs. NYHA II group and NYHA IV vs. NYHA III group. (C) The NT-proBNP concentration was positively correlated with the LAD (r=0.852, $\mathrm{P}<0.05)$. (D) The NT-proBNP concentration was negatively correlated with the $\mathrm{E} / \mathrm{A}(\mathrm{r}=-0.817, \mathrm{P}<0.05)$. ${ }^{*} \mathrm{P}<0.05$ vs. NYHA II group; ${ }^{* *} \mathrm{P}<0.01$ vs. control group; ${ }^{\#} \mathrm{P}<0.05$ vs. NYHA III group. NYHA, New York Heart Association classification; NT-proBNP, N-terminal pro-brain natriuretic peptide; LAD, left atrial diameter; E/A, ratio of the peak flow velocity in the early diastolic phase to the peak flow velocity in the late diastolic phase.
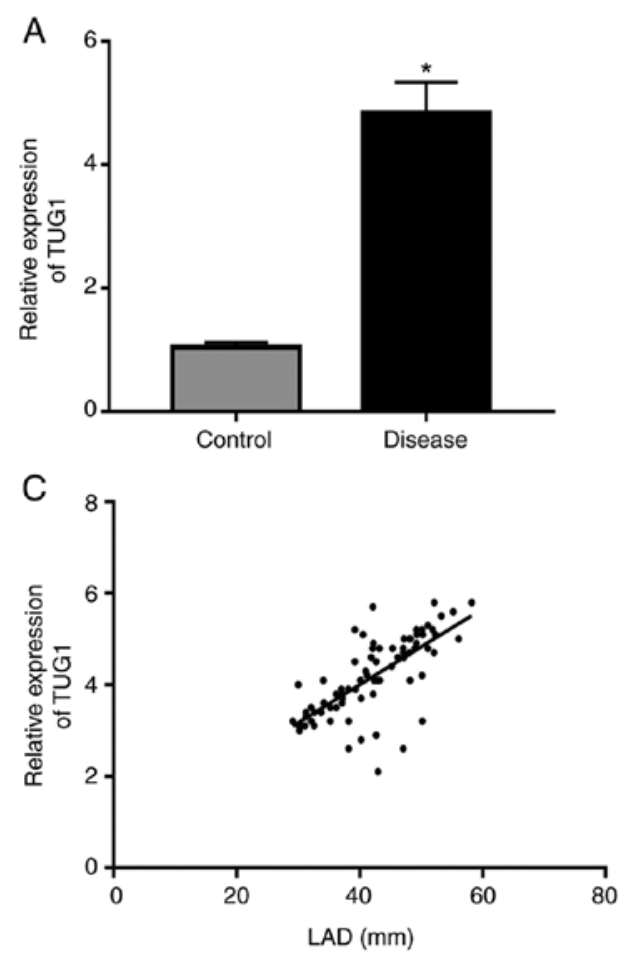

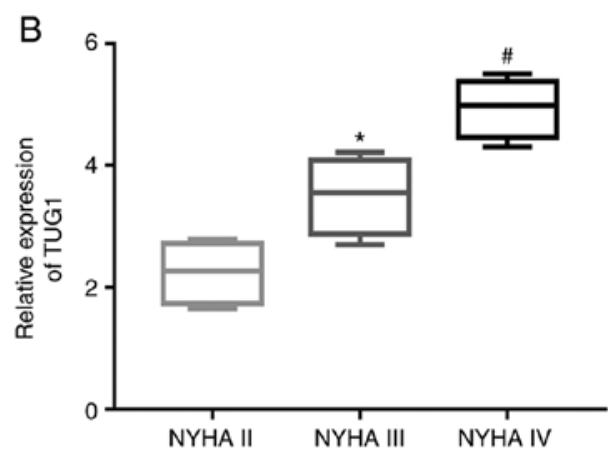

D

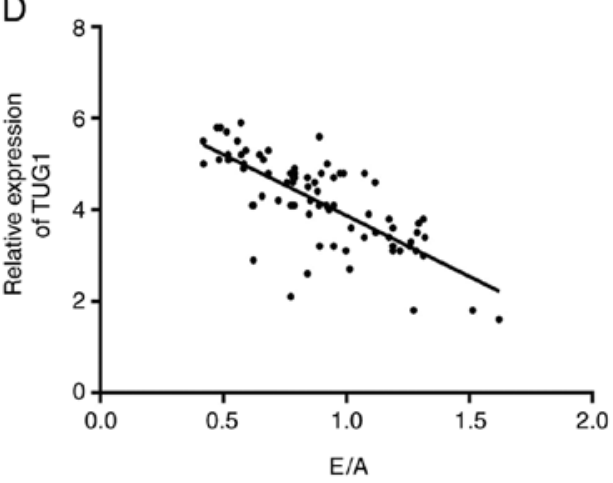

Figure 2. Serum levels of TUG1 are elevated in hypertensive patients with heart failure with preserved ejection fraction. (A) TUG1 was significantly enhanced in the observation group compared with that in the control group. Data were expressed as the mean \pm SD (B) TUG1 levels were significantly augmented in the NYHA III vs. NYHA II group and in the NYHA IV vs. NYHA III group. Data were expressed as median Q3-Q1. (C) TUG1 levels were positively correlated with the LAD ( $r=0.881, \mathrm{P}<0.05)$. (D) TUG1 levels were negatively correlated with the $\mathrm{E} / \mathrm{A}(\mathrm{r}=-0.871, \mathrm{P}<0.05)$. ${ }^{*} \mathrm{P}<0.05$ vs. NYHA II or control group; ${ }^{*} \mathrm{P}<0.05$ vs. NYHA III group. LAD, left atrial diameter; TUG1, taurine upregulated 1; NYHA, New York Heart Association classification; E/A, ratio of the peak flow velocity in the early diastolic phase to the peak flow velocity in the late diastolic phase. 
A

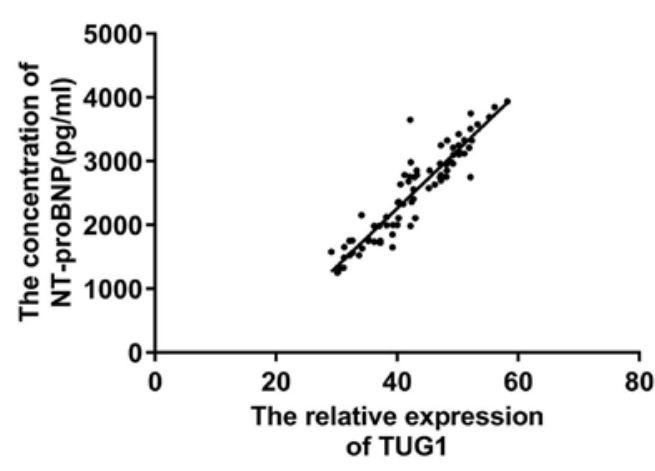

B

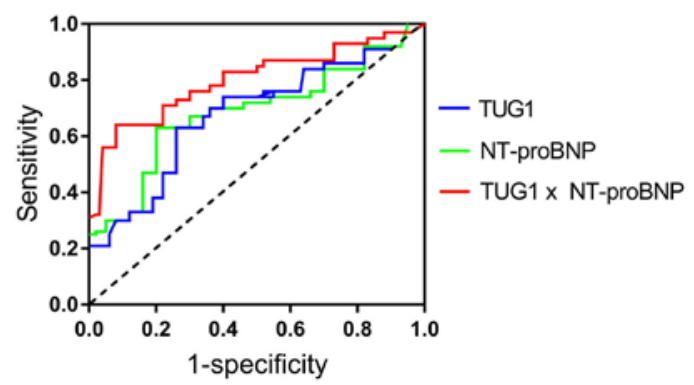

Figure 3. TUG1 and NT-proBNP may be useful biomarkers for the diagnosis of HFPEF in a hypertensive population. (A) A positive correlation was confirmed between the serum levels of TUG1 and NT-proBNP in hypertensive patients with hypertension and with heart failure with preserved ejection fraction $(r=0.823, \mathrm{P}<0.05)$. (B) Receiver operating characteristic curves for evaluating the ability of TUG1, NT-proBNP and their combination to identify patients with heart failure with preserved ejection fraction in a hypertensive population. TUG1 had an AUC value of 0.73 (95\% CI, 0.67-0.79; cut-off value, 0.43), NT-proBNP had an AUC value of 0.76 (95\% CI, 0.71-0.85 cut-off value, 0.45 ) and the combination of the two parameters had an AUC value of 0.83 (95\% CI, 0.74-0.89; cut-off value, 0.48). HFPEF, heart failure with preserved ejection fraction; NT-proBNP, N-terminal pro-brain natriuretic peptide; TUG1, taurine upregulated 1; AUC, area under the curve.

classification is the basis for the classification of the severity of clinical HF and the major criterion for judging the severity of the disease in the clinic (20). The present results indicated that NT-proBNP levels were increased with the severity degree of HF. Furthermore, Spearman's correlation analysis demonstrated the NT-proBNP concentration was positively correlated with the LAD and negatively correlated with the E/A. These data suggested that NT-proBNP may be used as one of the hematological diagnostic indexes for hypertensive patients with HFPEF.

As a novel lncRNA, TUG1 has been reported to participate in the development of cardiac diseases, including cardiac hypertrophy (21), ischemic myocardial injury (22) and atherosclerosis (23). Therefore, in the present study, the role of TUG1 in HF was investigated and it was determined that the serum levels of TUG1 in hypertensive patients with HFPEF were significantly higher than those in hypertensive controls. In addition, TUG1 levels were positively correlated with the LAD and negatively correlated with the E/A. A positive correlation was confirmed between the serum levels of TUG1 and NT-proBNP. Furthermore, ROC curve analysis revealed that the plasma levels of TUG1 and NT-proBNP are useful biomarkers for the diagnosis of HFPEF among hypertensive subjects and the combination of these two indexes slightly improved the diagnostic power.

In conclusion, the present study indicated that the NT-proBNP concentration and TUG1 expression levels were increased in the serum of hypertensive patients with HFPEF. Furthermore, TUG1 and NT-proBNP were determined to be useful plasma biomarkers for the diagnosis of HFPEF among hypertensive subjects.

\section{Acknowledgements}

Not applicable.

\section{Funding}

No funding was received.

\section{Availability of data and materials}

The datasets used and/or analyzed during the present study are available from the corresponding author on reasonable request.

\section{Authors' contributions}

RJ and BL performed the experiments. SZ designed experiments analyzed the data and wrote the manuscript. RJ and SZ can authenticate the raw data. All authors read and approved the manuscript.

\section{Ethics approval and consent to participate}

Written informed consent was provided by all study participants. The present study was approved by the ethics committee of The First Affiliated Hospital of Jinzhou Medical University (Jinzhou, China).

\section{Patient consent for publication}

Not applicable.

\section{Competing interests}

The authors declare that they have no competing interests.

\section{References}

1. Savarese G and Lund LH: Global public health burden of heart failure. Card Fail Rev 3: 7-11, 2017.

2. Benjamin EJ, Muntner P, Alonso A, Bittencourt MS, Callaway CW, Carson AP, Chamberlain AM, Chang AR, Cheng S, Das SR, et al: Heart disease and stroke statistics-2019 update: A report from the american heart association. Circulation 139: e556-e528, 2019.

3. Yancy CW, Jessup M, Bozkurt B, Butler J, Casey DE Jr, Drazner MH, Fonarow GC, Geraci SA, Horwich T, Januzzi JL, et al: 2013 ACCF/AHA guideline for the management of heart failure: A report of the American college of cardiology foundation/American heart association task force on practice guidelines. J Am Coll Cardiol 62: e147-e239, 2013.

4. Owan TE, Hodge DO, Herges RM, Jacobsen SJ, Roger VL and Redfield MM: Trends in prevalence and outcome of heart failure with preserved ejection fraction. N Engl J Med 355: 251-259, 2006. 
5. Paul B, Soon KH, Dunne J and De Pasquale CG: Diagnostic and prognostic significance of plasma N-terminal-pro-brain natriuretic peptide in decompensated heart failure with preserved ejection fraction. Heart Lung Circ 17: 497-501, 2008.

6. Geisler S and Coller J: RNA in unexpected places: Long non-coding RNA functions in diverse cellular contexts. Nat Rev Mol Cell Biol 14: 699-712, 2013.

7. Mattick JS and Rinn JL: Discovery and annotation of long noncoding RNAs. Nat Struct Mol Biol 22: 5-7, 2015.

8. Shi Q and Yang X: Circulating MicroRNA and long noncoding RNA as biomarkers of cardiovascular diseases. J Cell Physiol 231: 751-755, 2016.

9. Kumarswamy R, Bauters C, Volkmann I, Maury F, Fetisch J, Holzmann A, Lemesle G, de Groote P, Pinet F and Thum T: Circulating long noncoding RNA, LIPCAR, predicts survival in patients with heart failure. Circ Res 114: 1569-1575, 2014

10. Gao L, Liu Y, Guo S, Yao R, Wu L, Xiao L, Wang Z, Liu Y and Zhang Y: Circulating long noncoding RNA HOTAIR is an essential mediator of acute myocardial infarction. Cell Physiol Biochem 44: 1497-1508, 2017.

11. Wang F, Su X, Liu C, Wu M and Li B: Prognostic value of plasma long noncoding RNA ANRIL for in-stent restenosis. Med Sci Monit. 23: 4733-4739, 2017.

12. Young TL, Matsuda T and Cepko CL: The noncoding RNA taurine upregulated gene 1 is required for differentiation of the murine retina. Curr Biol 15: 501-512, 2005.

13. Ponikowski P, Voors AA, Anker SD, Bueno H, Cleland JGF, Coats AJS, Falk V, González-Juanatey JR, Harjola VP, Jankowska EA, et al: 2016 ESC Guidelines for the diagnosis and treatment of acute and chronic heart failure: The Task Force for the diagnosis and treatment of acute and chronic heart failure of the European Society of Cardiology (ESC)developed with the special contribution of the heart failure association (HFA) of the ESC. Eur Heart J 37: 2129-2200, 2016.

14. Elasfar A: Correlation between plasma N-terminal pro-brain natriuretic peptide levels and changes in New York Heart Association functional class, left atrial size, left ventricular size and function after mitral and/or aortic valve replacement. Ann Saudi Med 32: 469-472, 2012.
15. Livak KJ and Schmittgen TD: Analysis of relative gene expression data using real-time quantitative PCR and the 2(-Delta Delta C(T)) method. Methods 25: 402-408, 2001.

16. Bhargava S, Ali A, Manocha A, Kankra M, Das S and Srivastava LM: Homocysteine in occlusive vascular disease: A risk marker or risk factor. Indian J Biochem Biophys 49: 414-420, 2012.

17. Di Palo KE and Barone NJ: Hypertension and heart failure: Prevention, targets, and treatment. Heart Fail Clin 16: 99-106, 2020.

18. Fitzgibbons TP, Meyer TE and Aurigemma GP: Mortality in diastolic heart failure: An update. Cardiol Rev 17: 51-55, 2009.

19. Negi SI, Jeong EM, Shukrullah I, Raicu M and Dudley SC Jr: Association of low plasma adiponectin with early diastolic dysfunction. Congest Heart Fail 18: 187-191, 2012.

20. Poss J, Link A and Bohm M: Acute and chronic heart failure in light of the new ESC guidelines. Herz 38: 812-820, 2013 (In German).

21. Zou X, Wang J, Tang L and Wen Q: LncRNA TUG1 contributes to cardiac hypertrophy via regulating miR-29b-3p. In Vitro Cell Dev Biol Anim 55: 482-490, 2019.

22. Su Q, Liu Y, Lv XW, Dai RX, Yang XH and Kong BH: LncRNA TUG1 mediates ischemic myocardial injury by targeting miR-132-3p/HDAC3 axis. Am J Physiol Heart Circ Physiol 318: $\mathrm{H} 332-\mathrm{H} 344,2020$

23. Li FP, Lin DQ and Gao LY: LncRNA TUG1 promotes proliferation of vascular smooth muscle cell and atherosclerosis through regulating miRNA-21/PTEN axis. Eur Rev Med Pharmacol Sci 22: 7439-7447, 2018.

This work is licensed under a Creative Commons Attribution-NonCommercial-NoDerivatives 4.0 International (CC BY-NC-ND 4.0) License. 